\title{
Polarity Contributions to Hyperfine Splittings of Hydrogen-Bonded Nitroxides-The Microenvironment of Spin Labels
}

\author{
Derek Marsh \\ Abteilung Spektroskopie, Max-Planck-Institut für biophysikalische Chemie, 37070 Göttingen, Germany \\ E-mail: dmarsh@gwdg.de
}

Received March 5, 2002; revised May 16, 2002

\begin{abstract}
A self-consistent treatment of reaction field effects on isotropic ${ }^{14} \mathrm{~N}$ hyperfine coupling constants of nitroxide spin labels in mixtures of polar and apolar solvents is given based on the Onsager approach. It is shown that this works reasonably well for mixtures of water or methanol with dioxane, far better than do conventional approaches using the Clausius-Mossotti relation. Association constants, $K_{A, h}$, for hydrogen bonding of protic solvents to nitroxides are derived in this way from published EPR data. A value of $K_{A, h} \approx 1.0 \mathrm{M}^{-1}$ is argued to be reasonable for water in a hydrophobic environment. Data from spin-labelled lipids can then be used to estimate effective water concentrations in biological membranes. (c) 2002 Elsevier Science (USA)

Key Words: spin label; hyperfine splittings; solvent effects; polarity; dielectric polarization; hydrogen bonding.
\end{abstract}

\section{INTRODUCTION}

Currently there is considerable interest in the effects of hydrogen bonding on the spin Hamiltonian parameters of nitroxides (e.g., (1)). This arises primarily from the use of spin-label EPR to probe local water concentrations in biological systems (e.g., $(2,3)$ ). Isotropic ${ }^{14} \mathrm{~N}$ hyperfine couplings $a_{o}^{N}$, for instance, depend both on hydrogen bonding and on the local dielectric permittivity (4). The latter is related directly to the Onsager reaction field that varies according to $(\varepsilon-1) /(\varepsilon+1)$, where $\varepsilon$ is the dielectric constant. Determination of local proton donor (e.g., water) concentrations therefore requires allowance for the variation in effective local dielectric constant. In homogeneous mixtures of protic and aprotic solvents this may be done by direct measurement of the dielectric constant of the mixture (5). In heterogeneous systems, e.g., membranes and the interior of proteins, however, some estimate is needed of the effective local dielectric constant.

The purpose of this communication is to give a consistent treatment of the reaction field in solvent mixtures that is based on the Onsager result for the dielectric constant in multicomponent systems (6). This is then used to obtain association constants, $K_{A, h}$, for hydrogen bonding to nitroxides from EPR data. A previous approach based on the Clausius-Mossotti relation is not self-consistent and performs less well when compared with experimental dielectric constants (7). The Onsager treatment therefore may be used both to extract values of $K_{A, h}$ and to make polarity corrections in deriving local water concentrations from spin-label EPR data on biological systems. An example is given of water penetration in membranes.

\section{THEORETICAL BACKGROUND}

The isotropic ${ }^{14} \mathrm{~N}$-hyperfine coupling constant of a nitroxide in an aprotic solvent is given by (see $(4,6))$

$$
a_{o}^{N}(0)=a_{o, v}^{\varepsilon=1}+\frac{1}{2} K_{v}\left(n^{2}+2\right) \frac{\varepsilon-1}{2 \varepsilon+n^{2}},
$$

where $a_{o, v}^{\varepsilon=1}$ is the extrapolated isotropic coupling constant of the free nitroxide in a medium of dielectric constant $\varepsilon=1$, and $n$ is the refractive index of the nitroxide. To a good approximation, $n^{2} \approx 2.0$ for nitroxides (see, e.g., $(8)$ ). The strength of the reaction field, $K_{v}$, depends on the dipole moment and size of the nitroxide $(4,6)$.

In the presence of a proton donor, $\mathrm{PH}$, chemical exchange takes place between the free and hydrogen-bonded nitroxides, which have isotropic hyperfine couplings $a_{o}^{N}(0)$ and $a_{o, h}^{N}$, respectively. Al-Bala'a and Bates (7) showed that $a_{o, h}^{N}$ depends little on the dielectric constant of the medium. Assuming that exchange is fast compared with the difference in hyperfine couplings, the isotropic coupling constant observed experimentally is given by (see, e.g., (9))

$$
a_{o}^{N}([\mathrm{PH}])=\left(1-f_{h}\right) a_{o}^{N}(0)+f_{h} a_{o, h}^{N},
$$

where $f_{h}$ is the fractional population of hydrogen-bonded nitroxides. The latter is obtained from the law of mass action,

$$
f_{h}([\mathrm{PH}])=\frac{K_{A, h}[\mathrm{PH}]}{1+K_{A, h}[\mathrm{PH}]},
$$

where $K_{A, h}$ is the association constant for hydrogen bonding with the nitroxide. Combination of Eqs. [1]-[3] gives the 
following dependence of the isotropic ${ }^{14} \mathrm{~N}$-hyperfine coupling constant of a spin label on the concentration, $[\mathrm{PH}]$, of proton donor,

$$
a_{o}^{N}([\mathrm{PH}])=\frac{a_{o, v}^{\varepsilon=1}+K_{v} \frac{\varepsilon-1}{\varepsilon+1}+a_{o, h}^{N} K_{A, h}[\mathrm{PH}]}{1+K_{A, h}[\mathrm{PH}]},
$$

where $\varepsilon$ is the dielectric constant of the mixture of protic and aprotic solvents, which also depends on [PH].

The implicit result obtained by Onsager for the static dielectric constant, $\varepsilon$, in a multicomponent system is (6)

$$
\frac{\varepsilon-1}{\varepsilon}=\frac{1}{\varepsilon_{o}} \sum_{i}\left[\left(\frac{2+n_{i}^{2}}{2 \varepsilon+n_{i}^{2}}\right) \alpha_{i}+(2 \varepsilon+1)\left(\frac{2+n_{i}^{2}}{2 \varepsilon+n_{i}^{2}}\right)^{2} \frac{p_{i}^{2}}{9 k T}\right] N_{i},
$$

where $\alpha_{i}$ is the polarizability, $p_{i}$ is the electric dipole moment, $n_{i}$ is the refractive index, and $N_{i}$ is the number of molecules per unit volume, of component $i$, and $\varepsilon_{o}$ is the permittivity of free space. For comparison, the corresponding relation from Clausius and Mossotti as extended by Debye is

$$
\frac{\varepsilon-1}{\varepsilon+2}=\frac{1}{3 \varepsilon_{o}} \sum_{i}\left(\alpha_{i}+\frac{p_{i}^{2}}{3 k T}\right) N_{i}
$$

which is applicable strictly only to gaseous mixtures and is recovered approximately from Eq. [5] in the limit $\varepsilon-n_{i}^{2} \ll n_{i}^{2}$ (6). In Kirkwood's treatment for associated fluids, the second term on the right in Eq. [5], which describes the dipole contribution, is multiplied by a correlation parameter, $g_{i} \geq 1$. This extension allows for the mutual interactions of the dipoles and is a measure of their local ordering (10).

Consider a mixture of protic and aprotic solvents with dielectric constants $\varepsilon_{h}, \varepsilon_{v}$ and refractive indices $n_{h}, n_{v}$, respectively. It is assumed that the dipolar contribution to the dielectric constant of the protic solvent dominates over that of its polarizability, i.e., that $\alpha_{h} \approx 0$ in Eq. [5]. The aprotic solvent is taken to be apolar and hence its dipole moment is neglected; i.e., $p_{v} \approx 0$ in Eq. [5]. The dielectric constant of the mixture is then given by

$$
\frac{\varepsilon-1}{\varepsilon}=\frac{1}{\varepsilon_{o}}\left[(2 \varepsilon+1)\left(\frac{2+n_{h}^{2}}{2 \varepsilon+n_{h}^{2}}\right)^{2} \frac{p_{h}^{2}}{9 k T} \cdot N_{h}+\frac{2+n_{v}^{2}}{2 \varepsilon+n_{v}^{2}} \alpha_{v} N_{v}\right] .
$$

Application of Eq. [7] to the pure solvents in turn allows elimination of $p_{h}$ and $\alpha_{v}$. For $N_{v}=0: N_{h}=N_{h}^{o}$ and $\varepsilon=\varepsilon_{h}$; whereas for $N_{h}=0: N_{v}=N_{v}^{o}$ and $\varepsilon=\varepsilon_{v}$, where $N_{h}^{o}, N_{v}^{o}$ are the number densities of the pure solvents. For the mixture, evidently, $N_{h} / N_{h}^{o}=[\mathrm{PH}] /[\mathrm{PH}]_{o}$ and $N_{v} / N_{v}^{o}=1-[\mathrm{PH}] /[\mathrm{PH}]_{o}$, where $[\mathrm{PH}]_{o}$ is the molarity of the pure hydrogen-bonding solvent. Rewriting Eq. [7] then yields the following dependence of the dielectric constant on concentration $[\mathrm{PH}]$ of proton donor:

$$
\begin{aligned}
\frac{\varepsilon-1}{\varepsilon}= & \left(\frac{\varepsilon_{h}-1}{\varepsilon_{h}}\right)\left(\frac{2 \varepsilon+1}{2 \varepsilon_{h}+1}\right)\left(\frac{2 \varepsilon_{h}+n_{h}^{2}}{2 \varepsilon+n_{h}^{2}}\right)^{2} \frac{[\mathrm{PH}]}{[\mathrm{PH}]_{o}} \\
& +\left(\frac{\varepsilon_{v}-1}{\varepsilon_{v}}\right)\left(\frac{2 \varepsilon_{v}+n_{v}^{2}}{2 \varepsilon+n_{v}^{2}}\right)\left(1-\frac{[\mathrm{PH}]}{[\mathrm{PH}]_{o}}\right) .
\end{aligned}
$$

Expressing Eq. [8] in terms of the experimental dielectric constants largely compensates for the degree of approximation involved in the (in any case justifiable) assumptions made regarding the polarity of the two solvents. The expression given by Eq. [8] holds for Kirkwood's theory (10) only in so far as the correlation parameter, $g_{h}$, remains relatively constant, independent of concentration [PH]. Departures of $g_{h}$ from values for the neat hydrogen-bonding fluid are expected most at high dilution, for which one anticipates that $g_{h} \approx 1$. Under these conditions, Eq. [8] will overestimate the dielectric constant and the reaction field will saturate too rapidly.

\section{RESULTS}

\section{Polarity Contributions to Hyperfine Couplings}

Figure 1 gives the dependence of the isotropic hyperfine coupling constant, $a_{o}^{N}$, of 4-hydroxy-2,2,6,6-tetramethylpiperdine1-oxy (TEMPOL) on hydrogen donor concentration in mixtures of 1,4-dioxane with methanol or water (solid symbols) from the data of (5). Also included in Fig. 1 are estimates of the contribution to $a_{o}^{N}$ from the reaction field (open symbols) that were calculated by Gagua et al. (5) from interpolated values of the experimental dielectric constants for the solvent mixtures. Values of $a_{o, v}^{\varepsilon=1}=1.530 \mathrm{mT}$ and $K_{v}=0.035 \mathrm{mT}$ established with aprotic solvents are used in Eq. [1] for these estimates. This value of $K_{v}$ is low (and of $a_{o, v}^{\varepsilon=1}$ is high) compared with those reported for other nitroxides (e.g., $(4,7)$ ), because dioxane was used as the low-dielectric solvent in the calibration. It is known that values of $a_{o}^{N}$ in 1,4-dioxane are anomalously high-most probably caused by contributions from the dipole moment of the energetically unfavorable boat conformation (8). Nevertheless, this is the appropriate calibration for mixtures of protic solvents with dioxane.

The dashed lines in the lower part of each panel in Fig. 1 come from estimates of the reaction field that use Eq. [8] to obtain the dielectric constant. Values of $\varepsilon_{v}=2.209, n_{v}=1.4224$ for 1,4-dioxane, and of $\varepsilon_{h}=32.63$ (80), $n_{h}=1.3288$ (1.3328) for methanol (water) are used in these calculations. Onsager's treatment (i.e., Eq. [8]) represents the dielectric constant of the mixed solvents with a reasonable degree of accuracy, as seen from the correspondence between the dashed lines and open symbols. Deviations from the experimental values occur only at low concentrations, as was already anticipated. In contrast, predictions from the Clausius-Mossotti relation (Eq. [6]) are a rather poor approximation to the experimental estimates. These 


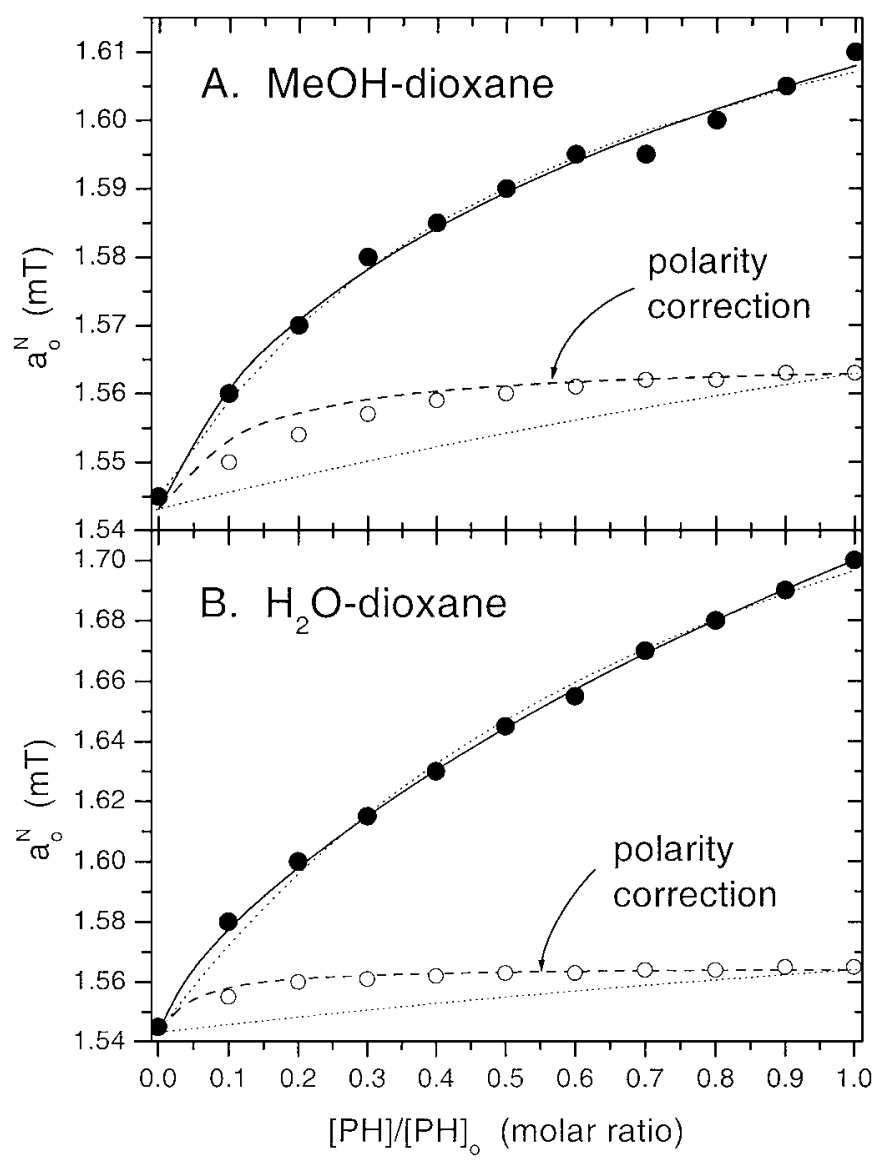

FIG. 1. Dependence of the isotropic hyperfine splitting constant, $a_{o}^{N}$, of 4-hydroxy-2,2,6,6-tetramethylpiperidine- $N$-oxy (TEMPOL) on proton donor concentration [PH], in mixtures with dioxane. (A) Methanol-dioxane mixtures. (B) Water-dioxane mixtures. Solid symbols are experimentally determined values of $a_{o}^{N}$. Open symbols are the polarity contributions to $a_{o}^{N}$ that are estimated from Eq. [1] by using experimental dielectric constants of the mixtures. Data from Gagua et al. (5). Solid line is the fit of Eqs. [4] and [8] to the experimental values of $a_{o}^{N}$ with the parameters given in the text. (The upper dotted line is the corresponding fit using the Clausius-Mossotti relation, instead of the Onsager expression.) In the lower part of each panel, the dashed line is the polarity contribution to $a_{o}^{N}$ obtained from Eq. [1] with the Onsager expression (Eq. [8]) for the dielectric constant and the dotted line is that with the Clausius-Mossotti relation (i.e., Eq. [6]).

are shown by the dotted lines in the lower part of each panel in Fig. 1. It is clear from this that corrections using the ClausiusMossotti relation are also far less significant than those based on the more realistic Onsager approach.

\section{Hydrogen-Bond Donors in Dioxane}

Nonlinear least squares fits of Eq. [4] to the experimental hyperfine couplings are given by the solid lines in Fig. 1. Using Eq. [8] to describe the dependence of the reaction field on proton donor concentration yields the following fitting parameters: $K_{A, h}=0.025 \pm 0.006 \mathrm{M}^{-1}, a_{o, h}^{N}=1.68 \pm 0.02 \mathrm{mT}$ for methanol, and $K_{A, h}=0.009 \pm 0.001 \mathrm{M}^{-1}, a_{o, h}^{N}=1.97 \pm 0.02 \mathrm{mT}$ for water. Fitting with the experimental estimates of the reaction field (i.e., the open symbols in Fig. 1) leads to optimized parameters that are quite close to the above: $K_{A, h}=0.039 \pm$ $0.007 \mathrm{M}^{-1}, a_{o, h}^{N}=1.65 \pm 0.01 \mathrm{mT}$ for methanol, and $K_{A, h}=$ $0.010 \pm 0.001 \mathrm{M}^{-1}, a_{o, h}^{N}=1.93 \pm 0.02 \mathrm{mT}$ for water. The Clausius-Mossotti relation, on the other hand, yields much larger deviations from this latter optimized parameter set: $K_{A, h}=0.073 \pm 0.010 \mathrm{M}^{-1}, a_{o, h}^{N}=1.631 \pm 0.005 \mathrm{mT}$ for methanol, and $K_{A, h}=0.019 \pm 0.002 \mathrm{M}^{-1}, a_{o, h}^{N}=1.82 \pm 0.02 \mathrm{mT}$ for water. These discrepancies lie outside the estimated range of uncertainty.

The values of $K_{A, h}$ for hydrogen donors in dioxane are rather small. Indeed, Gagua et al. (5) originally depicted the dependence of $a_{o}^{N}$ on [PH] as linear, with proportionality constant $K_{h}$. For $K_{A, h}[\mathrm{PH}] \ll 1$, Eq. [4] gives $K_{h} \approx\left(a_{o, h}^{N}-a_{o, v}^{N}\right) K_{A, h}$, where $a_{o, v}^{N}$ is the isotropic hyperfine constant in the aprotic solvent. This yields values of $K_{h} \approx 0.0042$ and $0.0040 \mathrm{mT} \cdot \mathrm{M}^{-1}$ for methanol and water, respectively. When the reaction field saturates quickly (i.e., for $\varepsilon \gg 1), K_{h} \approx\left(a_{o, h}^{N}-a_{o, v}^{\varepsilon=1}-K_{v}\right) K_{A, h}$ is a better approximation. This yields $K_{h} \approx 0.0034$ and $0.0038 \mathrm{mT} \cdot \mathrm{M}^{-1}$ for methanol and water, respectively. Whereas the concentration dependences for methanol and water are clearly nonlinear, those for ethanol and 2-propanol mixed with dioxane are not (5). For the latter, values of $K_{h} \approx 0.0024$ and $0.0028 \mathrm{mT} \cdot \mathrm{M}^{-1}$ are obtained, respectively, from linear fits. These values are quoted here for reference, and in consistent units, because of misprints in the original publication.

\section{Hydrogen-Bond Donors in Benzene}

In view of the far superior performance of the Onsager treatment, a reevaluation of hydrogen-bonding association constants derived using the Clausius-Mossotti approach is desirable. Figure 2 gives the data from (7) for the dependence of $a_{o}^{N}$ for 4-oxo-2,2,6,6-tetramethylpiperidine- $N$-oxy (TEMPONE) on proton donor concentration in mixtures of trifluoroethanol (TFE) or $n$-butanol with benzene. Nonlinear least squares fits of Eqs. [4] and [8] to the data for TFE and $n-\mathrm{BuOH}$ are given by the solid lines in Fig. 2. Fixed values of $a_{o, v}^{\varepsilon=1}=1.439 \mathrm{mT}$ and $K_{v}=0.0544 \mathrm{mT}$ are used in these fits (7). Polarity corrections to $a_{o}^{N}$ using these values in Eq. [1] are given by the dashed and dotted lines in the lower part of both panels in Fig. 2, for the Onsager and Clausius-Mossotti approaches, respectively. Table 1 gives the fitting parameters for TFE and $n-\mathrm{BuOH}$, along with those for several other proton donors. These values are obtained by using Eq. [8] from Onsager with the following dielectric constants: $\varepsilon_{h}=24.3,17.8,10.9,20.1,10.9,4.806$; and refractive indices, $n_{h}=1.2907,1.3993,1.3878,1.3850,1.5408,1.4459$, for TFE, $n-\mathrm{BuOH}, t-\mathrm{BuOH}, n-\mathrm{PrOH}, \mathrm{PhOH}$, and $\mathrm{CHCl}_{3}$, respectively; and $\varepsilon_{v}=2.284, n_{v}=1.5011$ for benzene. The ClausiusMossotti approach yields considerably different values for the fitting parameters, although the quality of the fit (given by the dotted lines in the upper part of each panel in Fig. 2) remains high. For $n$-BuOH the value of $K_{A, h}$ obtained with the 
TABLE 1

Association Constants, $K_{A, h}$, and Isotropic Hyperfine Coupling Constants, $a_{o, h}^{N}$, for Hydrogen-Bonding of Protic Solvents to TEMPONE in Benzene ${ }^{a}$

\begin{tabular}{lcccr}
\hline \multicolumn{1}{c}{$\mathrm{H}$-donor } & $K_{A, h}\left(\mathrm{M}^{-1}\right)$ & $a_{o, h}^{N}(\mathrm{mT})$ & $R$ & \multicolumn{1}{c}{$a_{o, n}^{N}(\mathrm{mT})$} \\
\hline $\mathrm{CF}_{3} \mathrm{CH}_{2} \mathrm{OH}$ & $1.01 \pm 0.07$ & $1.590 \pm 0.001$ & 0.9987 & $1.585 \pm 0.0025$ \\
$n$-BuOH & $0.48 \pm 0.07$ & $1.520 \pm 0.002$ & 0.9979 & $1.5150 \pm 0.0025$ \\
$t$ - $\mathrm{BuOH}$ & $0.74 \pm 0.13$ & $1.503 \pm 0.002$ & 0.9963 & $1.5012 \pm 0.0025$ \\
$n-\mathrm{PrOH}$ & $0.63 \pm 0.12$ & $1.507 \pm 0.002$ & 0.9971 & $1.505 \pm 0.0025$ \\
$\mathrm{PhOH}$ & $2.00 \pm 0.14$ & $1.595 \pm 0.001$ & 0.9997 & $1.590 \pm 0.0025$ \\
$\mathrm{CHCl}_{3}$ & $0.95 \pm 0.16$ & $1.491 \pm 0.001$ & 0.9934 & $1.4900 \pm 0.0025$ \\
\hline
\end{tabular}

${ }^{a}$ Deduced from the data of Al-Bala'a and Bates (7), by using Eqs. [4] and [8] (see text). $a_{o, n}^{N}$ is the isotropic hyperfine coupling constant in the neat solvent.

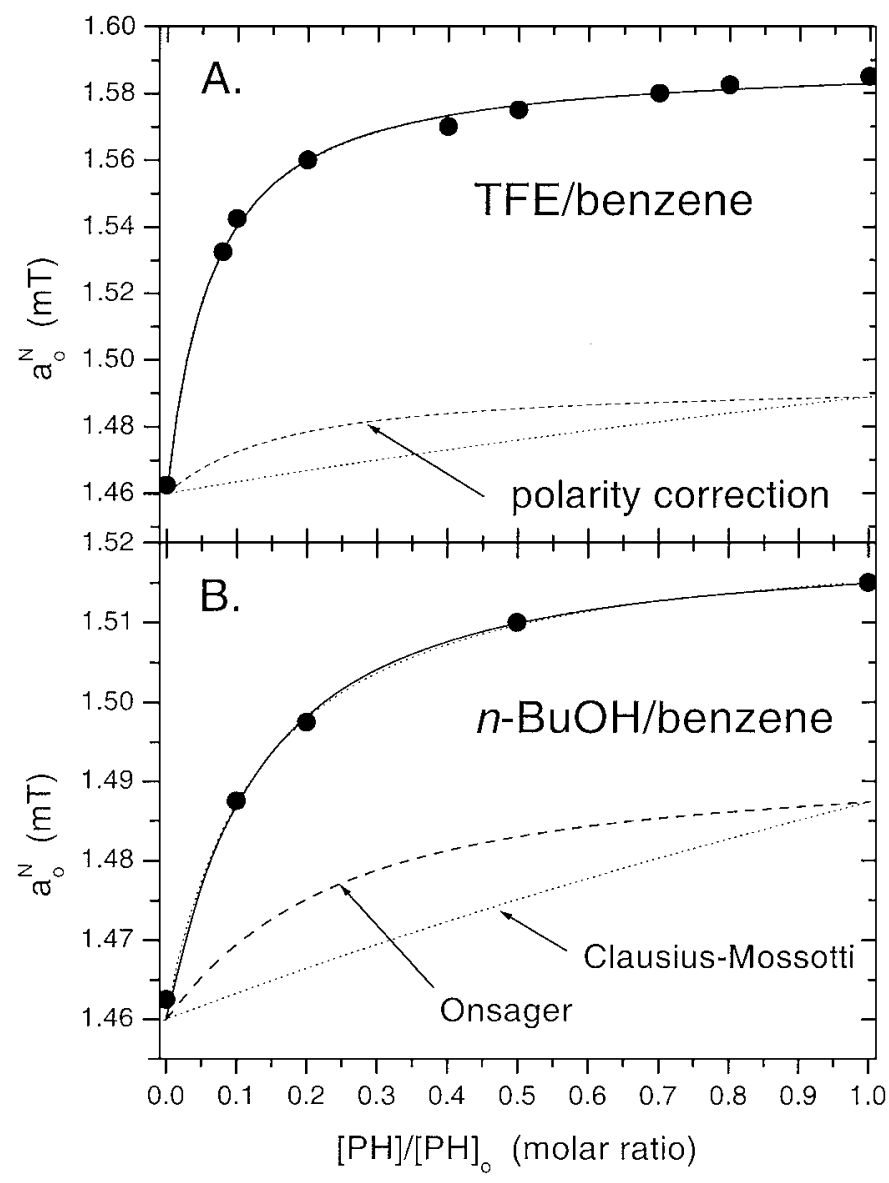

FIG. 2. Dependence of the isotropic hyperfine coupling, $a_{o}^{N}$, of 4-oxo2,2,6,6-tetramethylpiperidine- $N$-oxy (TEMPONE) on proton donor concentration $[\mathrm{PH}]$, in mixtures with benzene. (A) Trifluoroethanol (TFE)-benzene mixtures. (B) $n$-Butanol-benzene mixtures. Symbols are experimentally determined values of $a_{o}^{N}$. Data from Al-Bala'a and Bates (7). Solid line is the fit of Eqs. [4] and [8] to the experimental data with the parameters given in the text. (The upper dotted line is the corresponding fit using the Clausius-Mossotti relation, instead of the Onsager expression.) In the lower part of each panel, the dashed line is the polarity contribution to $a_{o}^{N}$ obtained from Eq. [1] with the Onsager expression (Eq. [8]) for the dielectric constant and the dotted line is that with the Clausius-Mossotti relation (i.e., Eq. [6]).
Clausius-Mossotti correction differs by $40 \%$ from that given in Table 1 , for $n-\mathrm{PrOH}$ by $50 \%$, for $t$ - $\mathrm{BuOH}$ by $20 \%$, and for TFE by $14 \%$. Only for $\mathrm{PhOH}(3 \%)$ and $\mathrm{CHCl}_{3}(1 \%)$ do the two values lie within the uncertainty range estimated from the fits.

\section{DISCUSSION}

The most striking aspect of the association constants for hydrogen bonding to the nitroxide is the very low value found for $\mathrm{H}$-donors in dioxane compared with those in benzene. Possibly this is related to the "anomalous" behavior of 1,4-dioxane as an apolar solvent that has been referred to already (cf. (8)). Presumably dioxane participates itself in hydrogen bonding with protic solvents. The values of $K_{A, h}$ that are given in Table 1 are therefore those most appropriate to spin labels in a hydrophobic environment. Severely limited miscibility precludes determinations for water, but the value of $K_{A, h}=1.0 \mathrm{M}^{-1}$ for TFE might be taken as representative because TFE has the highest dielectric constant in Table 1 and values of $a_{o, h}^{N}$ in TFE are reasonably close to those in water. The appropriate value of $a_{o, h}^{N}$ is that in pure water because those given in Table 1 lie very close to the values, $a_{o, n}^{N}$, in the neat hydrogen-bonding liquid.

With the above assumptions, Eqs. [4] and [8] may be combined to estimate effective local water concentrations from measurements of spin-label isotropic hyperfine coupling constants in biological systems. For the oxazolidine- $N$-oxy nitroxides used in spin labelling lipids, $a_{o}^{\varepsilon=1}=1.385 \mathrm{mT}, K_{v}=0.064 \mathrm{mT}$ (4), and $a_{o, h}^{N}=1.576 \mathrm{mT}$ in pure water. Hyperfine couplings in fluid bilayer membranes of dimyristoyl phosphatidylcholine are $a_{o}^{N} \approx 1.50 \mathrm{mT}$ in the upper part of the chain close to the polar headgroup region (viz., $\mathrm{C} 4-\mathrm{C} 7$ ), and $a_{o}^{N} \approx 1.44 \mathrm{mT}$ at the end of the chain near the center of the membrane (viz., C10-C14) (11). From Eqs. [4] and [8] this yields values of $\left[\mathrm{H}_{2} \mathrm{O}\right] /\left[\mathrm{H}_{2} \mathrm{O}\right]_{o}=0.019$ and $\varepsilon_{l o c}=3.10$ in the upper part of the chain, and $\left[\mathrm{H}_{2} \mathrm{O}\right] /\left[\mathrm{H}_{2} \mathrm{O}\right]_{o}=0.004$ and $\varepsilon_{l o c}=2.12$ in the middle of the membrane. In chromaffin granule membranes, corresponding hyperfine values are $a_{o}^{N} \approx 1.52 \mathrm{mT}$ and $1.40 \mathrm{mT}$ in the upper and terminal methyl regions of the chain, respectively (12). These then translate to values of $\left[\mathrm{H}_{2} \mathrm{O}\right] /\left[\mathrm{H}_{2} \mathrm{O}\right]_{o}=0.031$, 0.000 and $\varepsilon_{l o c}=3.92,1.87$ in the upper and middle regions of the membrane, respectively. Reference values of $\varepsilon_{v}=1.890$ and $n_{v}=1.3751$ appropriate to $n$-hexane were used for these calculations. Thus the center of chromaffin granule membranes is devoid of water, whereas a nonvanishing water concentration, equivalent to approximately $200 \mathrm{mM}$, is found in the middle of the hydrophobic region of dimyristoyl phosphatidylcholine bilayers. The high concentration of cholesterol in chromaffin granule membranes is the origin of this difference (2). It also causes the water concentration in the upper region of the chains to be higher in chromaffin granule membranes than in dimyristoyl phosphatidylcholine bilayers. The effective bulk water concentrations in this part of the membrane are in the region of 1-2 M. These are referred to as effective values because bulk solutions are used for calibration of surface concentrations in the 
two-dimensional membrane system. An appropriate alternative measure could be the water/lipid mole ratio, based on the above values of $\left[\mathrm{H}_{2} \mathrm{O}\right] /\left[\mathrm{H}_{2} \mathrm{O}\right]_{o}$ and the fact that the volume of a methylene group in a fluid membrane is comparable to that of $\mathrm{H}_{2} \mathrm{O}$ in liquid water (13). In principle, this could be extended to an effective surface concentration by using the area/lipid molecule (see, e.g., (13)).

\section{REFERENCES}

1. R. Owenius, M. Engström, M. Lindgren, and M. Huber, Influence of solvent polarity and hydrogen bonding on the EPR parameters of a nitroxide spin label studied by 9-GHz and 95-GHz EPR spectroscopy and DFT calculations, J. Phys. Chem. A 105, 10967-10977 (2001).

2. D. Marsh, Polarity and permeation profiles in lipid membranes, Proc. Natl. Acad. Sci. USA 98, 7777-7782 (2001).

3. H. J. Steinhoff, A. Savitsky, C. Wegener, M. Pfeiffer, M. Plato, and K. Möbius, High-field EPR studies of the structure and conformational changes of site-directed spin labeled bacteriorhodopsin, Biochim. Biophys. Acta 1457, 253-262 (2000).

4. O. H. Griffith, P. J. Dehlinger, and S. P. Van, Shape of the hydrophobic barrier of phospholipid bilayers: Evidence for water penetration in biological membranes, J. Membrane Biol. 15, 159-192 (1974).
5. A. V. Gagua, G. G. Malenkov, and V. P. Timofeev, Hydrogen-bond contribution to isotropic hyperfine splitting constant of a nitroxide free-radical, Chem. Phys. Lett. 56, 470-473 (1978).

6. L. Onsager, Electric moments of molecules in liquids, J. Am. Chem. Soc. 58, 1486-1493 (1936).

7. I. Al-Bala'a and R. D. Bates, Jr., Medium effects on ESR spectra in studies of hydrogen-bonded transient solvent-solute complexes, J. Magn. Reson. 73, 78-89 (1987).

8. A. H. Reddoch and S. Konishi, The solvent effect on di-tert-butyl nitroxide: A dipole-dipole model for polar solutes in polar solvents, J. Chem. Phys. 70, 2121-2130 (1979).

9. J. Gendell, J. H. Freed, and G. K. Fraenkel, Solvent effects in electron spin resonance spectra, J. Chem. Phys. 37, 2832-2841 (1962).

10. J. G. Kirkwood, The dielectric polarization of polar liquids, J. Chem. Phys. 7, 911-919 (1939).

11. P. Hoffmann, K. Sandhoff, and D. Marsh, Comparative dynamics and location of spin-labelled sphingomyelin and phosphatidylcholine in dimyristoyl phosphatidylcholine membranes studied by EPR spectroscopy, Biochim. Biophys. Acta 1468, 359-366 (2000).

12. P. Fretten, S. J. Morris, A. Watts, and D. Marsh, Lipid-lipid and lipidprotein interactions in chromaffin granule membranes, Biochim. Biophys. Acta 598, 247-259 (1980).

13. D. Marsh, "Handbook of Lipid Bilayers," CRC Press, Boca Raton, FL (1990). 